\title{
STUDY OF MIXED PHASE CLOUDS OVER WEST AFRICA: ICE-CRYSTAL CORNER REFLECTION EFFECTS OBSERVED WITH A TWO-WAVELENGTH POLARIZATION LIDAR
}

\author{
Igor Veselovskii ${ }^{1,2}$, Philippe Goloub ${ }^{3}$, Thierry Podvin ${ }^{3}$, Didier Tanre ${ }^{3}$, Albert Ansmann ${ }^{4}$, Michail \\ Korenskiy $^{1}$, Anatoli Borovoi ${ }^{5}$, Qiaoyun $\mathrm{Hu}^{3}$, Valentin Bovchaliuk ${ }^{3}$ David N. Whiteman ${ }^{6}$ \\ ${ }^{I}$ Physics Instrumentation Center, Moscow, Russia \\ ${ }^{2}$ Joint Center for Earth Systems Technology, UMBC, Baltimore, USA \\ ${ }^{3}$ Univ. Lille, Laboratoire d'Optique Atmosphérique, Lille, France \\ ${ }^{4}$ Leibniz Institute for Tropospheric Research, Leipzig, Germany \\ ${ }^{5}$ Institute of Atmospheric Optics, Tomsk, Russia \\ ${ }^{6}$ NASA GSFC, Greenbelt, USA
}

\begin{abstract}
Lidar sounding is used for the analysis of possible contribution of the corner reflection (CR) effect to the total backscattering in case of ice crystals. Our study is based on observations of mixed phase clouds performed during the SHADOW campaign in Senegal. Mie-Raman lidar allows measurements at $355 \mathrm{~nm}$ and 532 $\mathrm{nm}$ at $43 \mathrm{dg}$. off-zenith angle, so the extinction and backscattering Ångström exponents can be evaluated. In some measurements we observed the positive values of backscattering Ångström exponent, which can be attributed to the corner reflection by horizontally oriented ice plates.
\end{abstract}

\section{INTRODUCTION}

One of intriguing questions in the study of scattering phase function of ice crystals is the possible contribution of corner reflection (CR) effect to the total backscattering [1]. This socalled corner reflection may occur in case of ice crystals of simple shape, such as hexagonal plates and columns $[2,3]$. For example, if two faces of a crystal are perpendicular, they operate as a $2 \mathrm{D}$ corner reflector in backscattering directions. In contrast with specular reflection from horizontally oriented plates which occurs only for zenith pointing sounding geometry, the corner reflection from randomly oriented crystals may be predominant at any measurement angles [4].

The effects of diffraction and interference can be accounted for in the frame of physical-optics approximation [4]. Application of this model to the simplest case of randomly oriented hexagonal columns demonstrates that, in contrast with extinction coefficient which is spectrally independent, the account of Fraunhofer diffraction, associated with backscattered plane-parallel beams, results in spectrally dependent backscattering coefficient.

Corner reflection may occur also for oriented plates when they are probed by tilted lidars. Corresponding computations demonstrate that the effect is maximal when lidar is tilted at 30$40 \mathrm{dg}$ of zenith [5]. We should also keep in mind, that the corner reflection may be sensitive to the crystal deformation [5,6]. The surface roughness of crystal faces can also suppress the CR effect [1]. Thus there is a need of experimental data for better estimating the CR contribution to the backscatter of real ice clouds and, as previously mentioned, we are going to use the spectral dependence of the backscattering coefficient to identify the relevant cases.

In 2015-2016 the field campaign SHADOW was organized in Senegal in the framework of the CaPPA (Chemical and Physical Processed in the Atmosphere) project (http://www.labexcappa.fr/). The LILAS - Raman lidar used during the campaign, allows measurements of particle extinction, backscattering coefficient and depolarization ratio at $355 \mathrm{~nm}$ and $532 \mathrm{~nm}$. In our presentation we analyze their spectral dependence to evaluate the possible contribution of CR effect. 


\section{EXPERIMENTAL SETUP}

The instrumentation site is located at the IRD (Institute for Research and Development) Center, Mbour, Senegal $\left(14^{0} \mathrm{~N}, 17^{0} \mathrm{~W}\right)$. The observations were performed during MarchApril 2015 and December 2015-January 2016. Detailed information regarding the site and the instruments is reported in a recent publication [7]. The LILAS is based on a tripled Nd:YAG laser with a $20 \mathrm{~Hz}$ repetition rate, and pulse energy of $90 / 100 / 100 \mathrm{~mJ}$ at $355 / 532 / 1064 \mathrm{~nm}$. The aperture of the receiving telescope is 400 $\mathrm{mm}$. The lidar allows evaluation of three particle backscattering ( $355 \mathrm{~nm}, 532 \mathrm{~nm}, 1064 \mathrm{~nm})$ and two extinction coefficients $(355 \mathrm{~nm}, 532 \mathrm{~nm})$. To improve the system performance at $532 \mathrm{~nm}$, the rotational Raman channel was used instead of the vibrational one. The sounding was performed through an open window at a fixed off-zenith angle of $43 \mathrm{dg}$., which was the minimal possible angle, so the effect of specular reflection was excluded.

Depolarization measurements were performed at $532 \mathrm{~nm}$ only in March-April 2015 while depolarization data at $532 \mathrm{~nm}$ and $355 \mathrm{~nm}$ were both available for the December 2015 - January 2016 period. The relative uncertainty of depolarization measurements is estimated as $\pm 15 \%$ [7].

\section{RESULTS OF THE MEASUREMENTS}

Pure ice clouds occur mainly at altitudes where the atmospheric temperature is below the temperature of homogeneous water freezing (about $-40^{\circ} \mathrm{C}$ ), while at lower altitudes the ice crystals may coexist with super-cooled liquid water (SLW) droplets. During the SHADOW experiment we acquired about 170 hours of clouds observations in $5 \mathrm{~km} \mathrm{-} 14 \mathrm{~km}$ height range and, in approximately $25 \%$ of this time, SLW layers occurred. The liquid water layers were confined by the $10 \mathrm{~km}$ height.

A typical example of a mixed phase cloud observed during the 16-17 December 2015 night is shown in Fig.1. We provide the height-tempo distributions of range corrected lidar signal as well as the particle depolarization ratio at 532 nm. Between 22:00 and 23:40 UTC the backscattering coefficient at $532 \mathrm{~nm}$ presents strong enhancement inside the layer centered at approximately $9 \mathrm{~km}$ altitude. The depolarization ratio in this layer decreases, indicating the presence of SLW. The atmospheric temperature, measured by the radio-sonde in Dakar varies from $-28 \mathrm{C}$ at $8500 \mathrm{~m}$ to $-35 \mathrm{C}$ at $9500 \mathrm{~m}$, which are the heights corresponding to the bottom and to the top of the liquid layer observed in M'Bour. The SLW layer is observed for 1.5 hours and then dissipates. Below the SLW layer the particle depolarization ratio increases up to $\sim 40 \%$, indicating the presence of ice crystals. The optical depth of ice phase is significantly lower comparing to that of liquid one, because conversion of SLW droplets to ice crystals is associated to a size increase and a decrease of droplet number density.
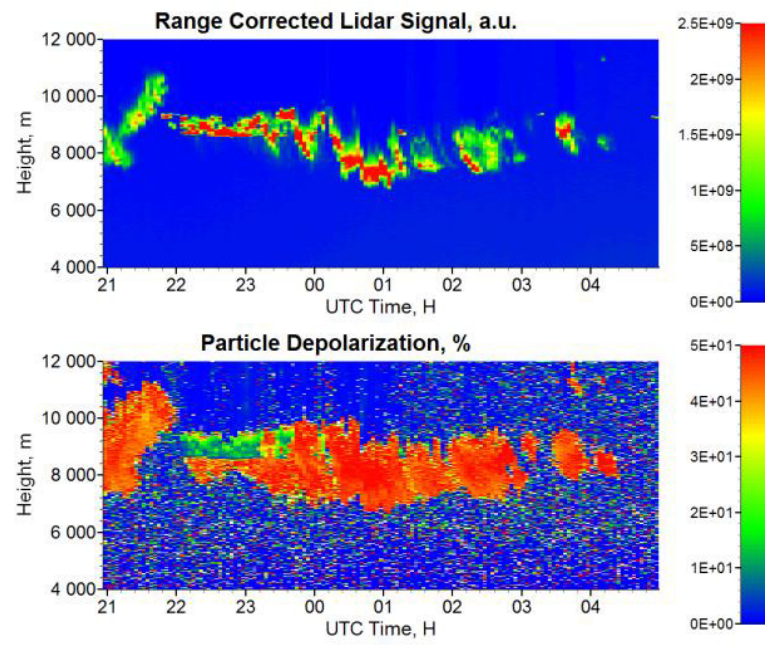

Figure 1. Range corrected lidar signal and (b) particle depolarization ratio at $532 \mathrm{~nm}$ measured in the 16-17 December 2015 night.

The vertical distributions of cloud backscattering coefficients and depolarization ratios at $355 \mathrm{~nm}$ and $532 \mathrm{~nm}$ are reported in Fig.2 for three time slots: 21:00-21:45 (pure ice cloud), 22:10-23:10 (liquid layer), and 00:00-01:00 UTC (ice cloud with residual SLW). The values of optical depth (OD) at $532 \mathrm{~nm}$ derived from Raman signals for these 3 periods are $1.49,1.41$ and 0.8 respectively. For the 21:00-21:45 time slot both particle depolarization ratios $\delta_{355}$ and $\delta_{532}$ are about $45 \pm 7 \%$ and do not present height dependence. The height averaged lidar ratio at both wavelengths is $22 \pm 4.5$ sr. Depolarization ratio in the SLW layer (second time slot 22:10$23: 10$ UTC) is low, $\delta_{532}$ is $8 \pm 1.5 \%$ at $8700 \mathrm{~m}$ 
(near the liquid layer base), still it may indicate the presence of ice crystals in liquid water surroundings. Above $9200 \mathrm{~m}$ the depolarization ratio increases, reaching $17 \%$ at $9400 \mathrm{~m}$ altitude. This increase of the depolarization ratio may be partly related to the contribution of multiple scattering. The averaged lidar ratios in liquid

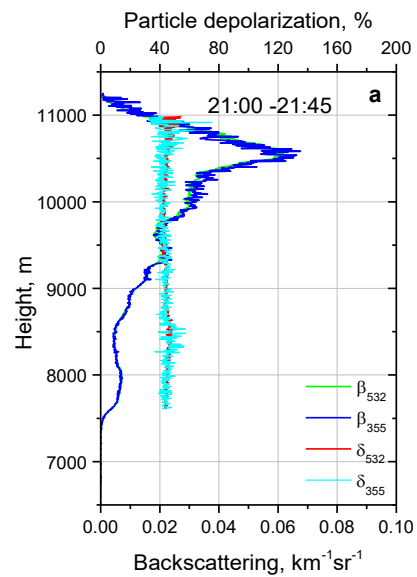

layer at both wavelengths are $15.5 \pm 3 \mathrm{sr}$, which is lower than in ice cloud. For the 00:00-01:00 time slot the depolarization ratio varies within the $25 \%-45 \%$ range due to residual SLW. The lidar ratio at both wavelengths is $17 \pm 3.5 \mathrm{sr}$, which corresponds to an intermediate value between ice crystals and liquid droplets.
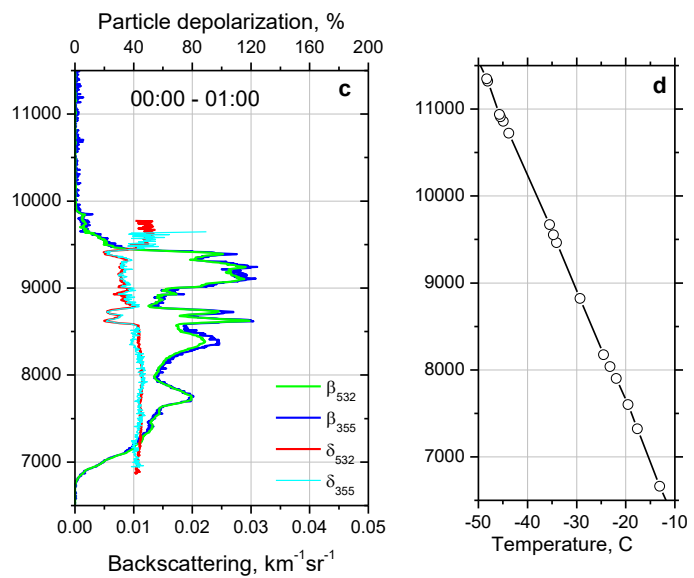

Figure 2. Vertical profiles of backscattering coefficients and particle depolarization ratios at 355 and $532 \mathrm{~nm}$. The measurements were performed in the night 16-17 December 2015 and results are averaged over the temporal interval (a) 21:00 - 21:45 UTC, (b) 22:10-23:10 UTC, (c) 00:00 - 01:00 UTC. Plot (d) shows temperature profile obtained from radio-sonde measurements in Dakar on 15 December at 12:00 UTC.
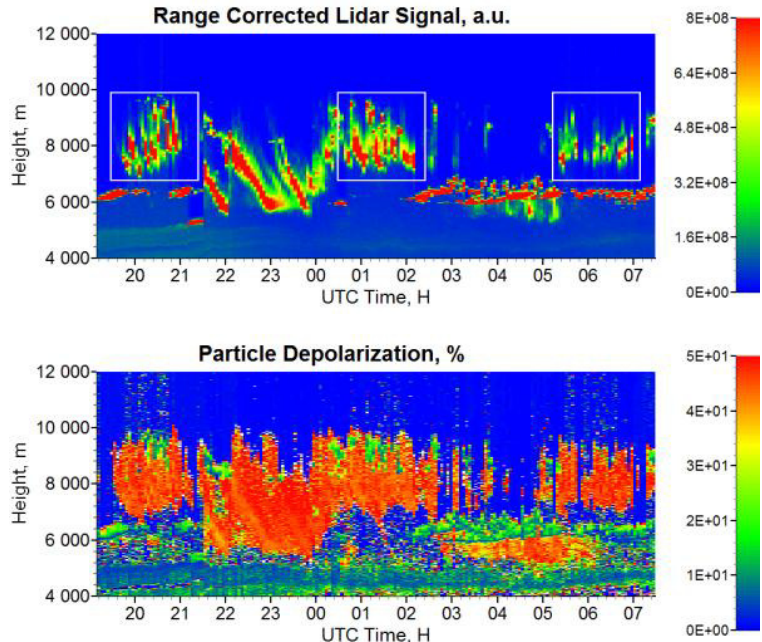

Figure 3. Range corrected lidar signal and particle depolarization ratio at $532 \mathrm{~nm}$ measured on 12-13 January 2016. White rectangulars mark the regions where spectral dependence of backscattering coefficient occurred.

For the three time slots, backscattering, extinction coefficients and depolarization ratios at $355 \mathrm{~nm}$ and $532 \mathrm{~nm}$ are close. No deviations of the backscattering Ångström exponent (BAE) from the zero value exceeding the measurement uncertainty (which we estimate to be below \pm 0.2 ), that could be attributed to the crystals corner reflection, are observed.

We have analyzed 15 representative measurement periods where ice clouds occurred and spectral dependence of backscattering coefficient was observed only on 12-13 January 2016. The heighttemporal distributions of range corrected lidar signal and depolarization ratio for that period are shown in Fig.3. The positive values of BAE were observed for time intervals 20:00-21:00, 00:30 02:30 and 5:30-7:00 UTC. For these intervals the lidar signals inside the $7000 \mathrm{~m}-9000 \mathrm{~m}$ height range are typical for ice precipitation, so presence of oriented ice crystals is possible.

Fig.4 shows the vertical profiles of backscattering coefficients $(\beta)$ and particle depolarization ratios $(\delta)$ at $355 \mathrm{~nm}$ and $532 \mathrm{~nm}$ averaged over 00:30 02:30 time period. Inside the height range 7000 $9000 \mathrm{~m} \beta_{355}$ exceeds $\beta_{532}$ and corresponding BAE reaches a maximal value of 0.6 at $8100 \mathrm{~m}$. Within the same height range the ratio $\delta_{355} / \delta_{532}$ decreases 
from 1.0 to 0.8 . The observed spectral dependence of backscattering coefficient and depolarization ratio can probably be explained by the corner reflection of oriented plates. The polarization planes of output laser radiation at $532 \mathrm{~nm}$ and 355 $\mathrm{nm}$ are orthogonal $(532 \mathrm{~nm}$ is vertical while 355 $\mathrm{nm}$ horizontal), so depolarization ratios of oriented plates in the case of corner reflection become different [5]. Similar spectral dependence of backscattering and depolarization was observed also for 20:00-21:00 period. The extinction coefficients $\alpha_{532}$ and $\alpha_{355}$ are close for all the considered height-temporal intervals. Thus the observed spectral dependence is attributed only to backscattering coefficients and depolarization ratios.
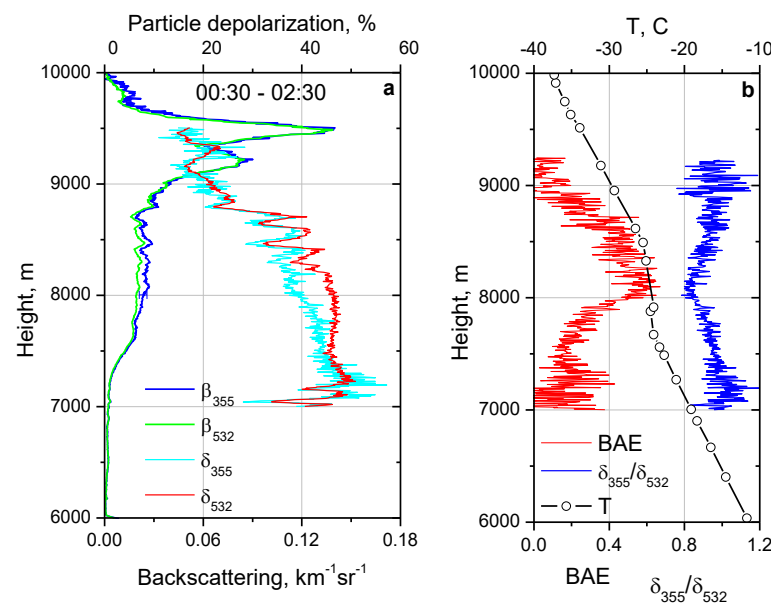

Figure 4. Vertical profiles of (a) backscattering coefficients and particle depolarization ratios at 355 $n m, 532 \mathrm{~nm}$, and (b) BAE together with ratio $\delta_{355} / \delta_{532}$ on 12-13 January 2016. Results of lidar measurements are averaged over 00:30 - 02:30 UTC period. Open symbols show temperature profile measured with radio-sounding in Dakar on 13 January at 00:00 UTC.

\section{CONCLUSIONS}

Most of the time, the contribution of the corner reflection to the total backscattering was not significant, but based on our knowledge, it is the first time that spectral dependence of backscattering coefficient, that can be related to the corner reflection of oriented ice plates, is observed. This low contribution of CR can be the result of crystals deformation or surface roughness. Another possible explanation can be the relative small amount of crystals of simple shape in the cases we selected.

\section{ACKNOWLEDGEMENTS}

Development of the software for lidar data analysis was supported by the Russian Science Foundation (project no.16-17-10241). The CaPPA project (Chemical and Physical Properties of the Atmosphere) is funded by the French National Research Agency (ANR) through the PIA (Programme d'Investissement d'Avenir) under contract "ANR-11-LABX-0005-01" and by the Regional Council " Nord-Pas de Calais FEDER".

\section{References}

[1] Zhou, C., and Yang, P: Backscattering peak of ice cloud particles, Opt. Expr. 23, 1199512003, 2015.

[2] Borovoi, A., Grishin, I., Naats, E., and Oppel, U.: Backscattering peak of hexagonal ice columns and plates, Opt. Lett. 25, 13881390, 2000.

[3] Del Guasta, M.: Simulation of lidar returns from ideal and deformed hexagonal ice prisms in cold cirrus by means of face tracing," J. Geophys. Res. 106, D12, 12,58912,602, 2001.

[4] Borovoi, A., Konoshonkin, A., and Kustova, N.: Backscattering by hexagonal ice crystals of cirrus clouds, Opt. Lett. 38, 2881-2884, 2013.

[5] Del Guasta, M., Vallar, E., Riviere, O., Castagnoli, F., Venturi, V., Morandi, M.: Use of polarimetric lidar for the study of oriented ice plates in clouds, Appl. Opt. 45, 48784887, 2006.

[6] Borovoi, A., Kustova, N., and Konoshonkin, A.: Interference phenomena at backscattering by ice crystals of cirrus clouds, Opt. Exp. 23, 24557-24571, 2015.

[7] Veselovskii, I., Goloub, P., Podvin, T., Bovchaliuk, V., Derimian, Y., Augustin, P., Fourmentin, M., Tanre, D., Korenskiy, M., Whiteman, D., Diallo, A., Ndiaye, T., Kolgotin, A., Dubovik, O.: Study of African dust with multi-wavelength Raman lidar during the "SHADOW" campaign in Senegal, Atm. Chem. Phys. 16, 7013-7028, 2016. 